\title{
Cycle Training Using Implanted Neural Prostheses: Team Cleveland
}

\author{
John McDaniel (1,2), Lisa M. Lombardo (1), Kevin M. Foglyano (1), Paul D. Marasco
} $(1,3)$, Ronald J.Triolo $(1,4)$

(1) Advanced Platform Technology Center, Louis Stokes Cleveland Veterans Affairs Medical Center, Cleveland, Ohio; (2)Exercise Science Program, Kent State University, Kent, Ohio; (3) Laboratory for Bionic Integration, Department of Biomedical Engineering, Lerner Research Institute, Cleveland Clinic, Cleveland, Ohio; (4) Biomedical Engineering, Case Western Reserve University, Cleveland, Ohio, USA

This article is distributed under the terms of the Creative Commons Attribution Noncommercial License (CC BY-NC 4.0) which permits any noncommercial use, distribution, and reproduction in any medium, provided the original author(s) and source are credited.

\begin{abstract}
Recently our laboratory team focused on training five individuals with complete spinal cord injuries for an overground FES bike race in the 2016 Cybathlon held in Zurich Switzerland. A unique advantage team Cleveland had over other teams was the use of implanted pulse generators that provide more selective activation of muscles compared to standard surface stimulation. The advancements in muscle strength and endurance and ultimately cycling power our pilots made during this training period helped propel our competing pilot to win gold at the Cybathlon and allowed our pilots to ride their bikes outside within their communities. Such positive outcomes has encouraged us to further explore more widespread use of FES overground cycling as a rehabilitative tool for those with spinal cord injuries. This review will describes our approach to this race including information on the pilots, stimulation strategy, bike details and training program.
\end{abstract}

Key Words: Spinal cord injury; Cybathlon; functional electrical stimulation; cycling; exercise

Eur J Transl Myol 27 (4): 289-294

Laboratories from around the world have put considerable effort into determining the efficacy of FES for those with spinal cord injuries. ${ }^{1,2}$ Historically our neuroprosthetic research program has focused almost exclusively on providing options for individuals with paralysis following spinal cord injury (SCI), stroke or multiple sclerosis (MS) to independently perform functional activities of daily living, such as standing to retrieve objects from overhead, ${ }^{3}$ transferring between seating surfaces of different heights, ${ }^{4}$ stabilizing the trunk and pelvis to improve wheelchair propulsion efficiency ${ }^{5}$ and facilitate bimanual reaching, ${ }^{6}$ and stepping short distances in the vicinity of the wheelchair to negotiate obstacles such as curbs and steps. ${ }^{7}$. Our entry into the 2016 Cybathlon in Zurich gave us motivation to refocus some of our efforts to training individuals for overground cycling.

\section{Pilot Details}

Our primary inclusion criteria for our pilots were to have an implanted neural prosthesis targeting muscles that would allow them to cycle. 5 of these individuals were enrolled to train for the Cybathlon with the intention of taking the top two to Zurich, one serving as a backup. In the end it was our backup pilot that actually competed in the race. The pilots trained ( 1 female, 4 males), ranged in age (45-59 years of age), level of injury (C7-T11) and time since injury (3-33 years). The duration in which they've had the implant also ranged from 2-32 years. These pilots followed the training program for several months and 2.5 months prior to the Cybathlon they competed against each other in a mock race which was used to determine which two pilots would travel to Zurich. The pilot that ultimately competed in the Cybathon was a male, 59 years of age with an 8.4 year old T4 AIS B injury. He has had his implant for 4.5 years and routinely takes oral baclofen (3x10mg daily) to minimize muscle spasticity.

\section{The race-ready bike}

The commercially available Catrike 700 recumbent tricycle was utilized as these tricycles have a lightweight aluminum frame bikes require a minimum of specialized parts and allowed for some minor modifications. The only neural interface specific modification to the tricycles was the addition of a US digital MA3-A10-236-N Miniature Absolute Magnetic Shaft Encoder (with a resolution of 0.35 
degrees) and bracket that monitored crank angle (Figure 1). The encoder gear was machined from aluminum and fit on the shouldered splines of the bottom bracket spindle. Crank angle information was sampled by the ECU, which modulated activation of the knee and hip extensor muscles appropriately to complete the pedaling motion. In all other aspects the tricycle was a normal bike. The team spent considerable time and energy reducing the weight and rolling resistance of these trikes which initially may seem over ambitious for a 750 meter flat course. However the team realized that by minimizing the rolling resistance and making the bikes as light as possible, our pilots would have a better chance of being able to cycle outdoors and that itself would likely improve adherence to the cycle training. The hand-built rear wheel was provided by Topolino Technology (Bethel, CT). It consisted of a carbon hub shell with aluminum free-hub body laced to a 700c alloy rim with carbon/kevlar composite spokes and aluminum alloy nipples; resulting in a slightly less than 800 gram wheel. The front wheels were stripped down and re-laced with Sapim (Antwerp, Belgium) CX-Ray spokes and 7000 series alloy nipples. Folding-clincher Schwalbe (Ferndale, WA) Pro One and Continental Grand Prix TT tires were chosen to reduce weight and rolling resistance and coupled with ultralight butyl rubber tubes instead of latex tubes to prevent air loss. The carbon arm TRP Spyre (Ogden, UT) SLC brake calipers were coupled to ultralight Ashima (Taichung City, Taiwan) Ai2 brake rotors with both wheel brakes routed to a single Paul Component Engineering (Chico, CA) duplex brake lever. This allowed the pilot to brake both wheels simultaneously with the left hand thereby freeing the right hand to manipulate the command buttons on the external control unit (ECU) to start and stop stimulation. All extraneous parts of the tricycles were removed including non-essential bearing seals, springs, pads, seat storage, padding, chainrings, front derailleurs, left shifters, and chainring protectors. Due to these modifications the weight of the primary racing bike was reduced from the stock $13.6 \mathrm{~kg}(30 \mathrm{lbs})$ to $11.8 \mathrm{~kg}(26 \mathrm{lbs})$. The freewheel drivetrain gear ratios were determined based on pedaling rates and power production (Garmin Vector 2 powermeter pedals; Garmin International, Olathe Kansas) that could be achieved across all the cogs in a gear cluster (cassette) while the trike was on a stationary trainer. In the end, a 42 tooth front chainring coupled with a 15/16 tooth cog allowed the riders to produce a pedaling rate (approximately $40 \mathrm{rpm}$ ) that maximized the power produced during a 45 second bout of cycling. Thus an 11-23 10-speed cassette was fitted to the bike as it placed the $15 / 16$ tooth $\operatorname{cog}$ at the center of the cassette range which allowed the riders to shift up or down if needed. To protect the insensate joints and reduce potential loss of power due to excess lateral movement and external rotation of the legs,

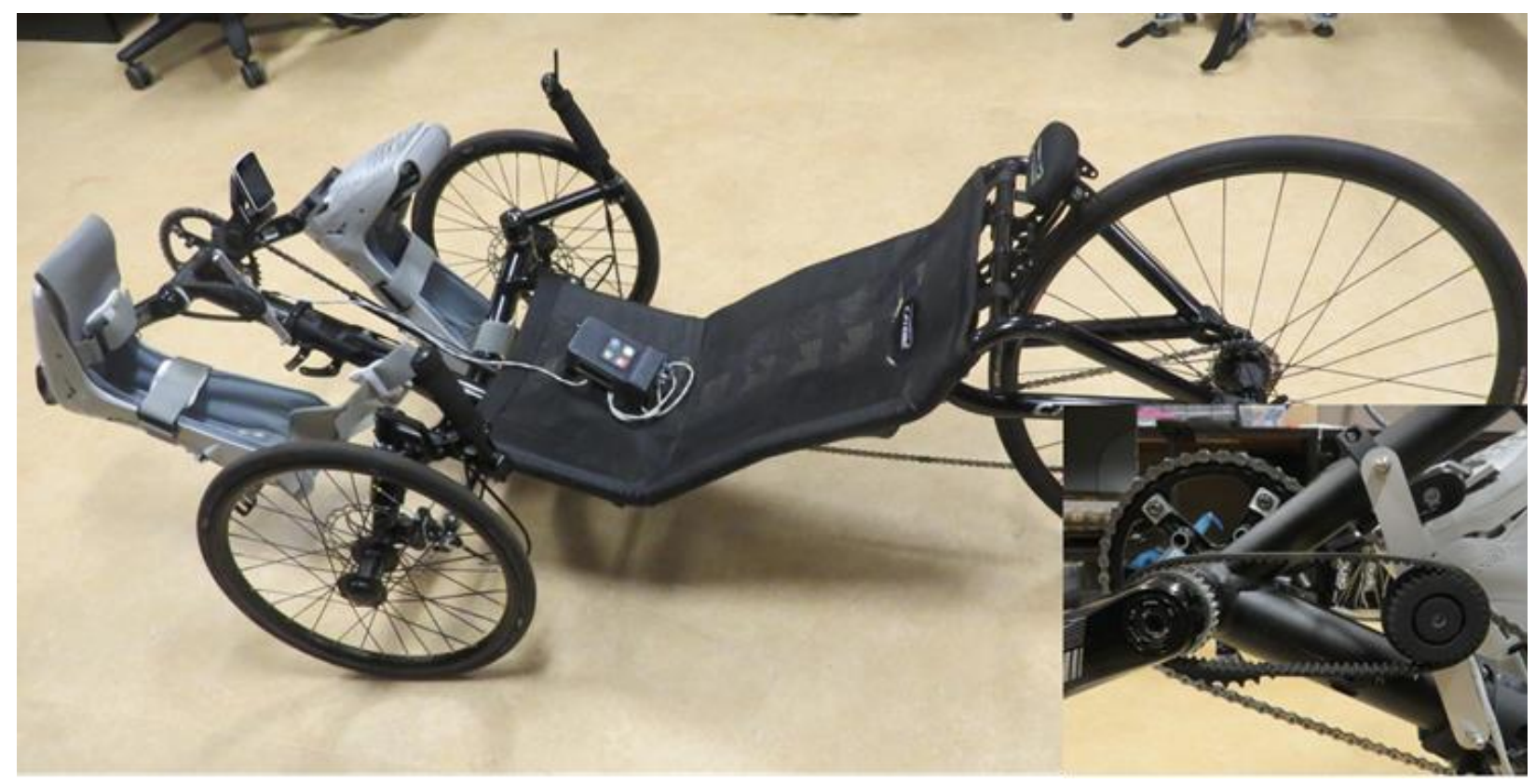

Fig 1. Picture of the modified trike with ECU on the seat. Note the ankle immobilizers were utilized to lock out the ankle. The insert illustrates the gear on the crank arm which is attached to the encoder that provides crank position to the ECU 
commercially available Aircast ankle-foot immobilizers were rigidly affixed to standard platform pedals just below the malleoli (Figure 1) to optimize force transfer and constrain non-sagittal hip motion.

\section{Electrical Stimulation}

Electrical stimulation for our FES cycling was achieved with a multichannel implantable pulse generators (IPGs) and muscle- and nerve-based electrodes our laboratory has developed over the past 3 decades. The implanted pulse generator (IPGs) are fabricated in the clean room facilities at Case Western Reserve University and implanted stimulating electrodes of our design were manufactured by Ardiem Medical, Inc (Indiana, PA USA). The system is approved by USFDA for investigational use only (IDE \#040214) and are currently available only in funded research studies taking place at Case and the Louis Stokes Cleveland VA Medical Center. These IPGs are surgically installed and can deliver 8, 12 or 16 independent channels of biphasic, charge-balanced current controlled stimulation of varying amplitude $(0.1-20 \mathrm{~mA})$, frequency $(1-50 \mathrm{~Hz})$ and pulse duration $(1-250 \mu \mathrm{sec})$. A wearable external control unit (ECU) transmits power and command information for various activation patterns wirelessly to the implants via a transcutaneous inductive link formed between a coil taped to the skin above the IPG and the implant. The ECU contains easily removable commercially available rechargeable Canon

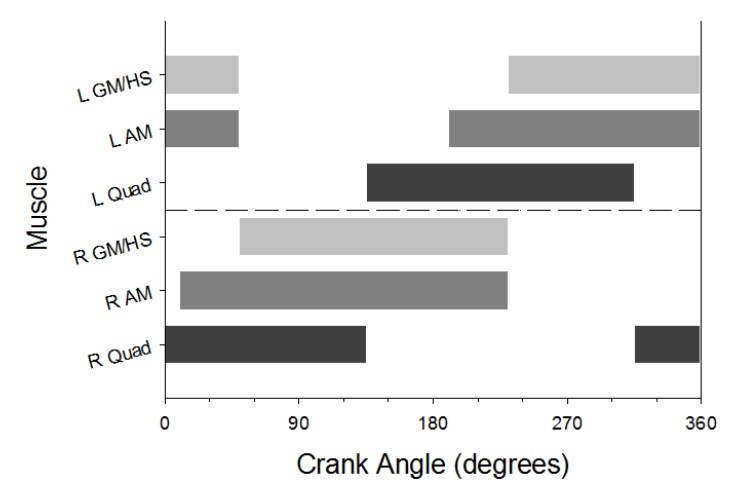

Fig 2. Stimulation patterns for the 5 pilots trained during this project. 0 degrees labels the position in which the right leg is fully flexed and begins to extend. At 180 degrees the left leg is fully flexed and begins to extend. Muscle abbreviations: $R$ and $L$ refer to right and left, Quad- quadriceps muscle group, $A M$ - posterior portion of adductor magnus, GM- gluteus maximus, HS- hamstrings (with an emphasis on semimembranosus). The grey scale is simply to separate muscle groups
7.2V1800mAh Li-ion batteries that can provide of 4-12 hours of continuous stimulation per charge (depending on stimulation parameters). The implanted system itself contains no batteries. Since the clinical implementation of the these systems (currently implanted in 30 individuals) was optimized for other research programs focusing on sitting stability, standing balance, stepping and seated reach, none of them specifically targeted all of the muscles required for the most efficient cycling.

Prior to the development of a training program, the team spent considerable time optimizing the stimulation parameters. The cycle activation patterns were based on the able-bodied and surface stimulation cycling literature, ${ }^{8,9}$, and ultimately tailored to the specific implanted muscle sets for each pilot. Fine tuning of stimulus timing and pulse parameters were guided by the crank position feedback from the encoder until smooth pedaling motions were achieved without any dead spots. In general, the muscles stimulated with the implants for all 5 pilots were the gluteus maximus, posterior portion of adductor magnus, quadriceps muscle group and semimembranosus. Spiral nerve cuff electrodes around the femoral nerves distal to the branching points to the sartorius and rectus femoris activate the uniarticular quadriceps for knee extension (vastus lateralis, medialis and intermedius). Stimulus amplitudes for the nerve cuff electrodes are adjustable up to $2 \mathrm{~mA}$. All other muscles were activated by intramuscular electrodes inserted near the nerve entry point at a current amplitude of $20 \mathrm{~mA}$. Stimulus pulse durations varied from 0 to 255 microseconds at a typical frequency of 20-25 hertz. Ultimately all five pilots utilized similar stimulation patterns (Figure 2). There was approximately 40 degrees of stimulation overlap between the right and left adductor magnus primarily to minimize hip abduction. To maximize speed, the team decided to take advantage of the large, unfatigued stimulated muscle output and improve the endurance of the muscles via a rigorous training regime. No automatic closed loop control of stimulus pulse parameters was attempted, only the timing of the muscle contractions to coincide with the stroke cycle. The pilot "closed the loop" by shifting gears to adjust for changing muscle output during the race.

\section{Training Information}

Prior to engaging in Cybathlon specific training, our 5 pilots had previous experience pedaling commercially available stationary surface stimulation exercise bikes as the team was successful in interfacing our implanted technology with those devices. These pilots also routinely performed other forms of exercise (ex. knee extension and standing) with their implanted systems. However, initial testing, revealed that our pilots fatigued much quicker than anticipated, producing approximately 30 watts or more for $15-20$ seconds before fatigue reduced power output to 10-15 watts for another minute. Thus, the training program developed maximized the 

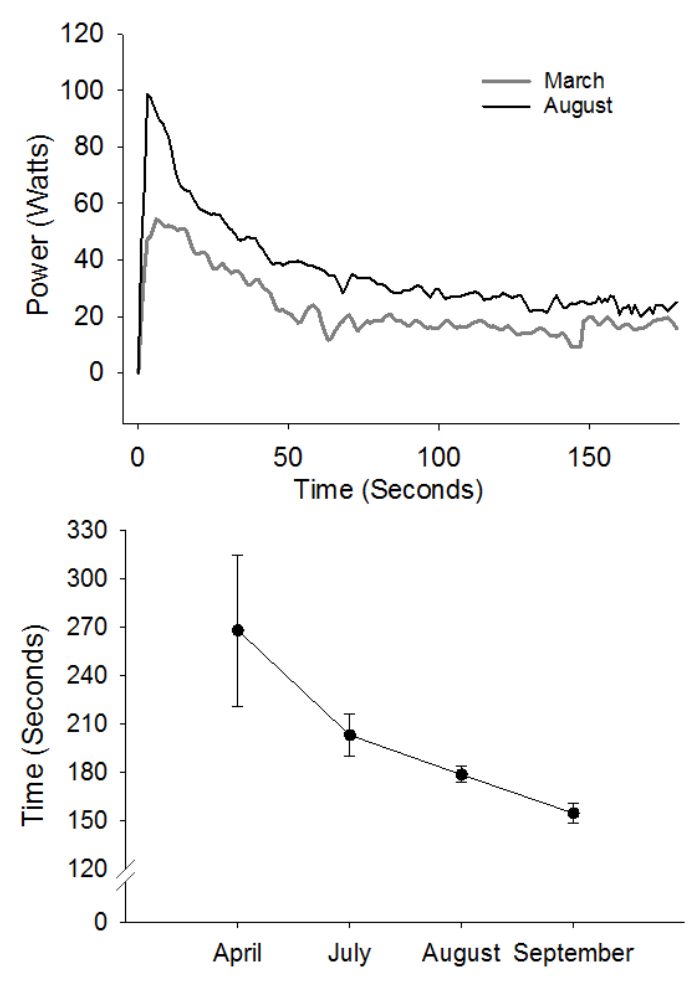

Fig 3. (Top) Power profile during a 3 minute bout of cycling in March and then again in August $(n=2)$. (Bottom ) Average time to complete 750 meters at 4 different timepoints throughout the training program $(n=2)$.

time they spent producing maximum power. The first protocol was a strength protocol which consisted of 60 seconds of stimulation followed by 30 seconds of rest. They were instructed to perform this in the most difficult gear ratio that would still allow them to complete these intervals for one hour. The second protocol, an endurance protocol, consisted of a 10minute bout of cycling followed by a 5-minute rest which they would repeat 4 times. At the start of the training program the pilots performed these protocols a combined 3-4 times per week on a stationary trainer with a little greater emphasis on the protocol that addressed their weakness (i.e., pilots with high power and low fatigue resistance focused on the endurance program and vice versa). There were two additional important aspects of our training program. First, training and race stimulation intensity was always set to maximize motor recruitment for the individual muscles. Reducing the stimulation intensity below max recruitment, which one might do for low intensity long duration training, would merely result in the primary recruitment of the fast twitch highly fatigable fibers and therefore incompatible with a long duration training session. Second, training was always performed on their trikes which forced them to produce power to spin the cranks rather than commercially available stationary FES bikes which have motors that engage to help the rider as they fatigue. We felt that stimulating fatigued muscles that are moving merely due to the assistance from a motor (passive movement) would not result in the same magnitude of physiological adaptations. The majority of the training took place at home on a stationary trainer as all five pilots were sent home with a bike, stationary trainer and an ECU for their implanted system programed with specific stimulation parameters for predetermined cycling workouts. Pilots were instructed to keep diaries of their training logs and the ECU monitored compliance. Initially the pilots followed the training program; however as they became stronger and less fatigable, they realized they could maintain the necessary power to cycle overground for extended periods of time and subsequently preferred to ride outdoors rather than on a stationary trainer.

\section{Performances}

Power output and $750 \mathrm{~m}$ time trials performances were routinely reexamined during the training period. This was valuable for the coaches and physiologists and also provided a source of motivation for the pilots. In the end 3 months of training did result in substantial improvements in the maximum power $(59 \pm 30$ watts to $102 \pm 46$ watts) and average power across three minutes ( $26 \pm 7$ watts to $42 \pm 3$ watts) for two pilots tested most frequently (Figure 3). These improvements in power resulted in substantial improvements in $750 \mathrm{~m}$ time trial performances $(4: 29 \pm 0: 55$ to $2: 35 \pm 0: 07)$. These times were also very consistent. During the visits they typically performed 3-4 time trials in which the finishing time would vary by only 5-10 seconds. As previously mentioned, a 750 meter mock race was used to determine which of the 5 pilots would go to Zurich. During this time trial the average finishing time for all 5 of our pilots was 3.46 and ranged from 3.07 to 4.49 . Beyond the standard training to improve fitness and muscular strength, another major focus was optimizing race day performance and recreating race conditions. Pilots simulated race conditions using a regulation size ramp to determine which gear to start with to take advantage of the early peak power output, to become efficient at shifting gears as they fatigued, and to practice switching lanes. During this time each pilot developed a sense of how much warm up time they needed for optimal performance. Furthermore, knowing the ventilatory response is delayed in this population our pilots were trained to mimic a normal ventilatory response by increasing breathing frequency from the start of each trial.

\section{Concluding Remarks}

One major difference between our pilots and every other pilot participating in the Cybathlon was the use of our implanted system that provided selective and repeatable activation of the targeted muscle groups. The major 
limitation is that these implants are not commercially available and require surgical procedure. It's also possible that a future infection could result in the removal of the implanted system. Currently the cost for the external control unit $(\$ 10 \mathrm{k})$, electrodes $(\$ 1000$ each $\mathrm{X} 16$ channels) and cost associated with the surgery is also a major limitation over traditional surface stimulation. Team Cleveland fully recognize and acknowledge that at this time utilization of surface stimulation is paramount to widespread dissemination and use of overground cycling by individuals with SCI, perhaps even in preparation for receiving an implanted system, and have recently started to more thoroughly compare the efficacy of using FES cycling with surface or implanted electrodes. Exchange of information with other teams present at the 2016 Cybathlon FES Cycling competition will provide further hints and tips. ${ }^{10-18}$ However, as there is very limited follow-up data on neuroprothesis beyond one year from implant, ${ }^{19}$ and the average duration from implant for our 5 pilots was 12 years, this project doubles as a long term follow-up study revealing the lasting benefits of these implants. The successful results from this project may foreshadow what the future holds for implantable neural prostheses.

\section{List of acronyms}

ECU - External Control Unit

IPG - Implantable Pulse Generators

MS - Multiple Sclerosis

SCI - Spinal Cord Injury

\section{Author's contributions}

All authors contributed to the writing of this manuscript and contributed to various aspects of this project. RT managed the project. JM, LL and KF developed the training program and worked with the pilots. PM modified the bike. RT, PM, LL and KF assisted the pilot at the Cybathlon.

\section{Acknowledgments}

We would like thank the following individuals for their support on this project: Musa Audu, J. Barber, E. Cikanek, A. Cornwell, D. Crago, J. French, V. Huynh, R. Kobetic, M. McClellan, J. Lee, M. Moynahan, M. Muhn, M. Nandor, S. Nogan-Bailey, B. Orzell, J. Schnellenberger. We would like to thank the following organizations for their support for this project: U.S. Department of Veterans Affairs - Rehabilitation Research and Development Service, Washington DC; Advanced Platform Technology Center - Louis Stokes Cleveland VA Medical Center, Cleveland, Ohio; Case Western Reserve University School of Engineering, Cleveland, Ohio; MetroHealth Medical Center Departments of Orthopedics and PM\&R, Cleveland, Ohio; University Hospitals - Department of Orthopedics, Cleveland, Ohio; NIH - National Institute of Neurological Disorders and Stroke, Bethesda, Maryland; Cleveland VA Medical Research and
Education Foundation, Cleveland, Ohio; Catrike, Orlando, Florida; City of Cleveland Heights; Center for Global Health Innovations, Cleveland, Ohio; Laboratory for Bionic Integration, Department of Biomedical Engineering, Lerner Research Institute, Cleveland Clinic, Cleveland, Ohio; Topolino Technologies, Bethel, Connecticut.

\section{Conflict of Interest}

There are no competing interests to report.

\section{Ethical Publication Statement}

Ethics approval and consent to participate. All procedures were approved the IRB at the Cleveland Louis Stokes Veterans Affairs Medical Center. All subjects signed consent to participate forms.

Consent for Publication Subjects provided their consent to publish their pictures and data.

We confirm that we have read the Journal's position on issues involved in ethical publication and affirm that this report is consistent with those guidelines.

\section{Corresponding Author}

John McDaniel, Exercise Science Program, Kent State University, 350 Midway Drive, Kent, Ohio 44242 Phone: 330-672-0802, Fax: 330-672-2250.

E-mail: jmcdani5@kent.edu

\section{E-mails of co-authors}

Lisa M. Lombardo: lisa.lombardo2@va.gov

Kevin M. Foglyano: kfoglyano@apt.org

Paul D. Marasco: marascp2@ccf.org

Ronald J.Triolo: Ronald.triolo@ case.edu

\section{References}

1. Guimaraes JA, da Fonseca LO, Dos Santos-CoutoPaz CC, et al. Towards Parameters and Protocols to Recommend FES-Cycling in Cases of Paraplegia: A Preliminary Report. Eur J Transl Myol 2016 Jun 13;26(3):6085. eCollection 2016 Jun 13.

2. Kern H, Carraro U. Home-Based Functional Electrical Stimulation for Long-Term Denervated Human Muscle: History, Basics, Results and Perspectives of the Vienna Rehabilitation Strategy. Eur J Transl Myol 2014;24:3296. doi: 10.4081/ ejtm.2014.3296. eCollection 2014 Mar 31.

3. Davis JA Jr, Triolo RJ, Uhlir J, et al. Preliminary performance of a surgically implanted neuroprosthesis for standing and transfers--where do we stand? J Rehabil Res Dev 2001;38:609-17.

4. Bieri C, Rohde L, Danford GS, et al. Development of a new assessment of effort and assistance in standing pivot transfers with functional electrical stimulation. J Spinal Cord Med 2004;27:226-35.

5. Triolo RJ, Bailey SN, Lombardo LM, et al. Effects of intramuscular trunk stimulation on manual wheelchair propulsion mechanics in 6 subjects with spinal cord injury. Arch Phys Med Rehabil 


\section{Cycle Training Using Implanted Neural Prostheses}

Eur J Transl Myol 27 (4): 289-294

2013;94:1997-2005. doi: 10.1016/ j.apmr.2013. 04.010. Epub 2013 Apr 26.

6. Wu GA, Lombardo L, Triolo RJ, Bogie KM. The effects of combined trunk and gluteal neuromuscular electrical stimulation on posture and tissue health in spinal cord injury. PM R 2013;5:688-96.

7. Triolo RJ, Bieri C, Uhlir J, et al. Implanted Functional Neuromuscular Stimulation systems for individuals with cervical spinal cord injuries: clinical case reports. Arch Phys Med Rehabil 1996;77:1119-28.

8. Hunt KJ, Ferrario C, Grant S, et al. Comparison of stimulation patterns for FES-cycling using measures of oxygen cost and stimulation cost. Med Eng Phys 2006 ;28:710-8. Epub 2005 Nov 18.

9. Roth N, Wiener A, Mizrahi J. Methods for Dynamic Characterization of the Major Muscles Activating the Lower Limb Joints in Cycling Motion. Eur J Transl Myol 2014;24(3):3317. doi: 10.4081/ejtm.2014.3317. eCollection 2014 Sep 23.

10. Coste CA, Mayr W, Bijak M, et al. FES in Europe and Beyond: Current Translational Research. Eur J Transl Myol 2016;26(4):6369.

11. Azevedo Coste C, Bergeron V, Berkelman R, et al. Comparison of strategies and performance of functional electrical stimulation cycling in spinal cord injury pilots for competition in the first ever CYBATHLON. Eur J Transl Myol 2017;27:251-4.

12. Berkelmans R, Woods B. Strategies and performances of Functional Electrical Stimulation Cycling using the BerkelBike with Spinal Cord Injury in a competition context (CYBATHLON). Eur J Transl Myol 2017;27:255-8.
13. Laubacher M, Aksöz EA, Bersch I, Hunt KJ. The road to Cybathlon 2016 - Functional electrical stimulation cycling Team IRPT/SPZ. Eur J Transl Myol 2017;27:259-64.

14. Sijobert B, Fattal C, Daubigney A, AzevedoCoste B. Participation to the first Cybathlon: an overview of the FREEWHEELS team FES-cycling solution. Eur J Transl Myol 2017;27:265-71.

15. Guimarães JA, Oliveira da Fonseca L, de Sousa AC, et al. FES Bike Race preparation to Cybathlon 2016 by EMA team: a short case report. Eur J Transl Myol 2017;27:272-78.

16. Metani A, Popovic-Maneski L, Mateo S, et al. Functional electrical stimulation cycling strategies tested during preparation for the First Cybathlon Competition - a practical report from team ENS de Lyon. Eur J Transl Myol 2017;27:279-88.

17. Arnin J, Yamsa-ard T, Triponyuwasin P, Wongsawat $Y$. Development of practical functional electrical stimulation cycling systems based on an electromyography study of the Cybathlon 2016. Eur J Transl Myol 2017;27:295301.

18. Leung WC, Tong RKY, Wang X, et al. The Effectiveness of Functional Electrical Stimulation (FES) in On-Off Mode for enhancing the cycling performance of Team Phoenix at 2016 Cybathlon. Eur J Transl Myo 2017;27:302-6.

19. Guiraud D, Azevedo Coste C, Benoussaad M, Fattal C. Implanted functional electrical stimulation: case report of a paraplegic patient with complete SCI after 9 years. J Neuroeng Rehabil 2014;11:15. doi: 10.1186/1743-0003-1115 . 